\title{
Custom Process to Small Business
}

\author{
Rodrigo Rocha Silva \\ FATEC-SP \\ CISUC \\ Mogi das Cruzes, Brasil \\ rodrigo.rsilva@fatec.sp.gov.br
}

\author{
Fernanda Yuri Kimura \\ FATEC-SP \\ Mogi das Cruzes, Brasil \\ feerkimura@hotmail.com
}

\author{
Jorge Bernardino \\ Polytechnic of Coimbra \\ CISUC \\ Coimbra, Portugal \\ jorge@isec.pt
}

\author{
Joubert de Castro Lima \\ UFOP \\ Ouro Preto, Brasil \\ joubert@iceb.ufop.br
}

\begin{abstract}
In this work we customized the RUP process with Scrum practices, and proposed a differentiate trace ability matrix, applying in a small company. The experimental results show that our customization can be adopted as an alternative to a systematic and less-intrusive process.
\end{abstract}

Keywords - Software Development Process, Scrum, RUP

\section{INTRODUCTION}

Some practitioners and academics have proposed different process that combines two or more methods $[1,2]$. The objective of these approaches was to create a process that maximized the strengths of the involved methods while at the same time reducing their weaknesses to improve the software development lifecycle and produce high-quality products [1].

The main goal of our custom process is to guarantee a constant delivery routine of software artifacts that have business value. In order to validate our custom process, we present the results of its adoption in a small software company.

\section{OUR CUSTOM PROCESS}

Our custom process fulfills all the basic premises of software development processes, such as iteractions and verifications with delivery forecast of mandatory artifacts.

The customization proposed is strongly focused on design and de velopment, suggesting only the following RUP artifacts: I. DVS or technical proposal for high-level definition of the scope and purpose of the system; II. Mapping of functional requirements; non-functional; and business rules; for specification of functionalities, presenting the constraints, validations, and exceptions that the system must obey; III. Description of functionalities; IV. Software Domain Mapping.

Our custom process defines three roles similar to the Scrum roles, for which each description can be found below: I. Product owner; II. Guardian; III. Team. Our custom process is divided into three phases, just like in the Scrum, although it receives the name of the RUP phases: Initiation, Elaboration and Construction, that compose the development Sprints and the Transition phase.

In order to ensure the control of requirement changes, our custom process suggests the creation of a traceability matrix. The implementation of test units with the greatest possible coverage is a key practice in using our custom process.

\section{RESULTS}

Our custom process was applied in a real project of Company X, where typically, the projects are developed by a team of 3 to 5 people. Prior to our custom process application, the software development process was based on informality, without documentation of requirements and scheduling of the next meetings. Most of the times, deadlines were not met and there were many changes in the requirements during the development phase because those were not previously foreseen due to the lack of planning during the specification and analysis processes.

As proposed by our custom process, the control of the project was carried out by implementing the culture of sending weekly reports, thus maintaining a communication between the team and the project manager.

In order to validate whether the requirements were developed according to the specification, the developer himself performed the tests on the functionalities developed, which were later validated by the systems analyst. The inconsistencies found were documented by the systems analyst and sent to the developer for correction.

In relation to the schedule, it was estimated four months to end the project. Tasks were completed as planned only in the first two sprints. The last three weeks were delayed by two weeks each, which represents $30 \%$ of the total planned. The low number $(5 \%)$ of changes in requirements was also evident.

The adoption of the reporting culture was also one of the new habits that faced difficult adaptation. Resistance to new practices was the greatest difficulty at implementing our custom process.

\section{CONCLUSIONS}

The implementation of our custom process was carried out with a compatible cost to small companies. At first, the change of culture generated dissatisfaction in the development team, and it was of great importance their awareness that a defined process is fundamental for the improvement of the final product and also makes the work of all the involved ones, easier.

Future works intend to apply our custom process in other companies as well as in different projects, besides inserting alternatives to the practices for projects with differentiated scopes.

\section{REFERENCES}

[1] Nortier, B., K. Von Leipzig, and C. Schutte, "The Development of a Software Development Framework by Combining Traditional \& Agile Methods to Address Modern Challenges," ISEM 2011 Proceedings, September 21-23, Stellenbosch, South Africa, 2011.

[2] Bashir, M. S. and M. R. J. Qureshi, "Hybrid Software Development Approach for Small to Medium Scale Projects: RUP, XP \& SCRUM," Science International (Lahore) 24, 4 (2012), pp. 381-384 